for about 80 days - or $9 \%$ - longer than control mice, with females surviving 20\% longer. The animals were also less likely to develop certain signs of ageing, such as loss of insulin sensitivity. The gene-expression patterns of the mice were similar to those in mice undergoing long-term caloric restriction, suggesting that manipulating S6K1 signalling could be a strategy for researchers seeking drugs that mimic the positive effects of this regime.

\title{
STEM-CELL BIOLOGY
}

\section{Rebooting cord blood cells}

\section{Cell Stem Cell 5, 434-441; 353-357 (2009)}

A well-known cocktail of genes can reset many adult cells to 'pluripotency', a state from which they can develop into almost any tissue. Now, two groups have derived induced pluripotent stem (iPS) cell lines from umbilical cord blood, a source that could be clinically useful.

Ulrich Martin of Hannover Medical School in Germany and his colleagues created the cells from cord blood using four genes, OCT4, SOX2, NANOG and LIN28. Juan Carlos Izpisúa Belmonte at the Salk Institute for Biological Studies in La Jolla, California, and his collaborators generated iPS cells using as few as two genes, OCT4 and SOX2.

Cord-blood cells have not acquired as many mutations as other cells, so stem cells such as these might be less prone to turn cancerous if used as therapy.

Nevertheless, both groups used viruses to insert the genes, reducing the cells' direct therapeutic utility.

\section{JOURNAL CLUB}

\section{Judith E. Mank}

Edward Grey Institute, Department of Zoology, University of Oxford, UK

An evolutionary biologist compares genomic complexity to modern art.

Like many students of evolutionary biology, I was taught that genes encode physical traits, or 'phenotypes', that are the focus of natural selection - a model with clear, direct links and few, if any, complications. Over the past few years, I have found it

\section{MICROBIOLOGY}

\section{Bacteria fight back}

J. Exp. Med. doi:10.1084/jem.20090097 (2009)

A bacterium that causes many skin and bloodborne infections and the bacterium responsible for anthrax both synthesize the same molecule to evade host immune responses.

Olaf Schneewind and his colleagues at the University of Chicago in Illinois examined the ability of Staphylococcus aureus (pictured below) to survive in rodent blood. They found that an enzyme anchored to the bacterium's cell wall produces adenosine, a key signalling molecule, during infection to protect the bacteria from attack by white blood cells. The anthrax pathogen Bacillus anthracis uses the same mechanism for survival.

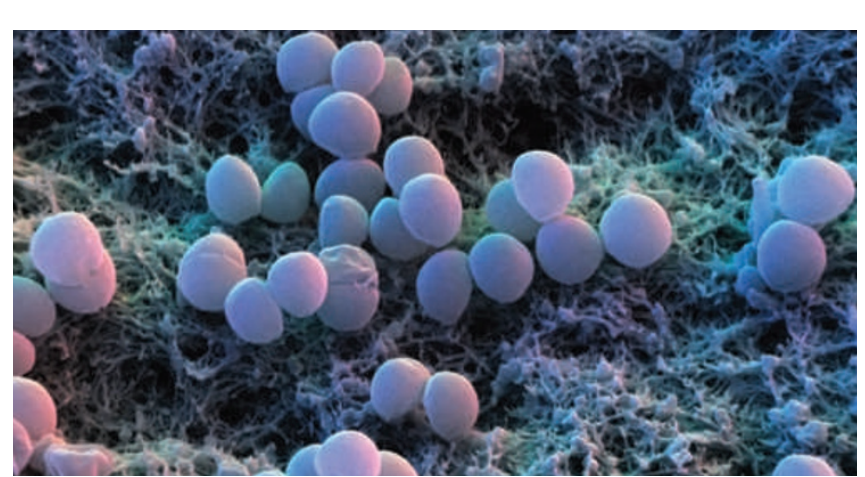

CHEMISTRY Microwave magic

Angew. Chem. Int. Edn doi:10.1002/anie.200904185 (2009)

Microwave irradiation is commonly used to boost the speed and yield of chemical reactions, but how it works has been unclear. Debate centres on whether it is due to the heat supplied or to some effect of microwaves' electromagnetic field on the reaction components.

Oliver Kappe and his colleagues at Karl Franzens University in Graz, Austria, have separated the two effects using silicon carbide vials, which transmit the heat but block out their electromagnetic field.

When they measured reaction time and product yield in 18 microwave-enhanced reactions, the researchers obtained almost identical results with silicon carbide vials as with Pyrex containers. This suggests that in most cases heat is responsible for the benefits of microwave chemistry.

\section{MATERIALS SCIENCE}

\section{No gas from glass}

Nature Mater. doi:10.1038/nmat2542 (2009)

Where temporary surgical implants are concerned, materials that decompose safely over time eliminate the need for costly and painful removal. Magnesium in its crystalline form has been used in some devices because it is about as strong as bone. But when it corrodes, it releases hydrogen gas, raising the risk of gas pockets being formed in tissues.

Jörg Löffler and his colleagues at the Swiss Federal Institute of Technology in Zurich sharply reduced gas release by creating glassy magnesium-zinc alloys. The gas reduction - which took place only if the alloy's zinc content was at least $28 \%$ - happened because a dense outer layer of zinc oxide or carbonate forms when the magnesium in these alloys decomposes, preventing hydrogen from forming bubbles. increasingly difficult to reconcile this simple model connecting genes and the organisms they encode with the burgeoning data of systems biology, which show the genome as a heaving tangle of interconnections. Given the complexity of the genome, how can selection target any single gene without unintended consequences?

Trudy Mackay at North Carolina State University in Raleigh and her collaborators have begun to resolve the opposing genomic and evolutionary world views by examining the systems genetics that underlie phenotypes in the fruitfly Drosophila melanogaster
(J. F. Ayroles et al. Nature Genet. 41, 299-307; 2009). They do this by comparing data on the abundance of more than 10,000 DNA transcripts with whole-organism traits, such as fitness and lifespan, in 40 fruitfly lines.

The researchers show that aggregates of genes correlate with distinct characteristics in flies, and that these modules are connected, with groups of genes associated with multiple phenotypic traits. This elegant complexity is best conveyed by the figures in the paper, some of which look as though they were lifted off the walls of a modern-art gallery.
The group's work provides a post-genomic framework for dissecting the intricate underpinnings of organismal biology. More importantly, the paper demonstrates that key topics in traditional evolutionary studies, such as heritability, and more recent concepts, such as pleiotropy (whereby one gene affects multiple traits), are related. As such, they must be considered together to build a complete understanding of how selection acts through the phenotype to sculpt the genome.

Discuss this paper at http://blogs. nature.com/nature/journalclub 